\title{
"Because of Them I will Never Give up": Perspective of Patients Receiving Specialist Palliative Care in the Kumasi Metropolis, Ghana
}

\section{Gladys Anyane*, Rasheed Ofosu-Poku, Michael Owusu-Ansah and Alberta Delali Dzaka}

Directorate of Family Medicine, Komfo Anokye Teaching Hospital, Kumasi, Ghana

*Corresponding Author: Gladys Anyane, Directorate of Family Medicine, Komfo Anokye Teaching Hospital, Kumasi, Ghana.
Received: September 15, 2021

Published: September 22, 2021

C) All rights are reserved by Gladys Anyane., et al.

\begin{abstract}
The global need for palliative care continues to grow as a result of the rising burden of non-communicable diseases and an ageing population. Each year, an estimated 40 million people are in need of palliative care. The aim of palliative care is to improve the quality of life of individuals and families who are faced with life-threatening illness, and to offer a support system which helps patients live as actively as possible until death. This study aims at discovering the perspective of patients receiving specialist palliative care at the Komfo Anokye Teaching Hospital. A qualitative approach with exploratory design was employed. Using purposive sampling, ten (10) participants were recruited and individual face-to-face in-depth interviews were conducted. Interviews were audio-recorded, transcribed verbatim, and thematic analysis done. Two (2) themes and seven (7) subthemes were identified. Patients appreciated being respected as individuals and involved in decision making, as well as the receptiveness of team members, their compassion, and the assistance they offered in achieving good symptom control. They also highlighted the cost-effectiveness of palliative care services, its team approach to care, and the home-visit service it offers. All clinicians must therefore integrate the principles of basic palliative care in their daily practice and advocate referral when needs of patients get complex, to improve their quality of life.
\end{abstract}

Keywords: Perception of Patient; Palliative Care; Communication; Quality of Life; Cancer

\section{Abbreviations}

KATH: Komfo Anokye Teaching Hospital; WHO: World Health Organization

\section{Introduction}

Chronic non-communicable diseases cause $71 \%$ of deaths annually, accounting for 41 million deaths each year [1]. Thus, an estimated 40 million people will require palliative care annually, but only $14 \%$ of them receive it [2]. Majority of patients who do not receive palliative care services despite their needs are found in low- and middle-income countries such as Ghana where there is an estimated $80 \%$ of the global need for palliative care [3].

In Ghana, palliative care began during the 1970s at some sickle cell clinics, then in 2003 at Ripples Health Care. Palliative care was started as a specialist service in August 2012 when it was officially started at the Korle-Bu Teaching Hospital [4] and Tetteh Quashie Memorial Hospital in 2016 as a specialist service [5]. In 2015, a specialist palliative care service was commenced at the Komfo Anokye Teaching Hospital (KATH) to augment the care of patients with chronic conditions and help improve their quality of life. The 
palliative care team at KATH offers out-patient and in-patient specialist consultations.

Since the inception of palliative care at KATH, knowledge about the palliative care team's achievement of the objective of improving patients and families' quality of life and the perspective of patients and families on the impact of the palliative care service remains unknown. Moreover, according to Sampson, Finlay, Byrne, Snow and Nelson (2018), the perspective of patients on palliative care is fundamental to the provision, practice, and evaluation of optimal care. The voice of patients can help in the future improvement of palliative services as their perspective of what is important in their care represents an aspect of quality palliative care [6].

A study on the perspective of patients on palliative care was therefore necessary to enable palliative care providers identify the impact of their services and note areas requiring improvement. This study therefore explores patients' perspective of specialist palliative care at KATH.

\section{Materials and Methods}

Study design and setting

A qualitative approach and exploratory design was used for this study as it focuses on gaining insight into the perspective of the patients [7-9]. KATH is the second largest hospital in Ghana and has a specialist palliative care service running under the Family Medicine Directorate. Participants for this study were recruited from the palliative care outpatient clinic.

\section{Study population and sampling}

The study population comprised of outpatients receiving palliative care from the palliative care team. Participants included in the study were those who had received palliative care for a minimum of two weeks. Patients who are unable to communicate verbally or had severe cognitive impairments that prevents them from understanding questions and engaging in meaningful conversations were excluded from the study.

Purposive sampling method was used to recruit the participants. The sample size consisted of nine (9) participants, as determined by the point of data saturation when no new findings or information emerged.

\section{Data collection and handling}

A semistructured interview guide was employed for data collection. The instrument was pretested with two participants to ensure clarity of the guiding questions. Data obtained from the pretest was not added to the study.

Contact numbers of potential participants were obtained from records available from the palliative care unit. All other potential participants were contacted by phone and those who expressed interest in participating met in person for further explanation about the study.

Face-to-face in-depth interviews were done and interviews audio-taped with the permission of participants. Each interview lasted for an average of 30 minutes and were conducted from $7^{\text {th }}$ to $28^{\text {th }}$ May, 2019. The data generated was transcribed verbatim, manually coded and analyzed thematically.

Signed consent forms, hard copies of transcripts, and audio-recording of interviews on an external drive have been safely kept in a locked cabinet for at least five years. All electronic data have been saved in a folder and password protected.

\section{Methodological rigor}

Interview guide was pretested among two participants and necessary modification was made to ensure that relevant data was collected. Debriefing sessions were held to ensure that the description of the data collected was accurate. Thick description of the study setting to provide adequate background information about the participants as well as the research context was given to ensure transferability of study findings. An audit trial of field notes, transcripts, audio records, interview questions, and consent forms has been kept for future confirmatory purposes.

\section{Ethics statement}

Ethical clearance was obtained from the Committee on Human Research Publication and Ethics of Kwame Nkrumah University of Science and Technology, Kumasi.

\section{Findings}

\section{Demographic characteristics}

Patients who participated in the study were aged between 32 years and 56 years, had all been diagnosed of a metastatic cancer, and consisted of 5 males and 5 females. 
Themes and sub-themes

The themes (2) and subthemes (7) from the transcribed data have been presented below in table 1 .

\begin{tabular}{|l|c|}
\hline Themes & Sub-Themes \\
\hline \multirow{4}{*}{ Structure of care } & Multidisciplinary team approach \\
\cline { 2 - 2 } & Consultation setting \\
\cline { 2 - 2 } & Preferred model of care \\
\cline { 2 - 2 } Process of care & Cost effectiveness \\
\hline \multirow{3}{*}{} & Communication \\
\cline { 2 - 2 } & Involvement in decision making \\
\cline { 2 - 2 } & Symptom management \\
\hline
\end{tabular}

Table 1: Themes and subthemes from transcribed data.

\section{Structure of care}

This theme discusses the views of patients on how they perceived palliative care services to be structured. Emerging subthemes were the team approach, consultation setting, cost effectiveness of care, and the availability of a preferred model of care.

\section{Multidisciplinary team approach}

Participants, depending on their needs, had received some form of care from other specialists coordinated by the palliative care team. Discussing the employment of a multidisciplinary team approach to meeting a need, one participant said: The palliative care physician referred me with a letter to see the ENT specialist because I had difficulty swallowing (Participant 1).

Another participant, who was sad due to his disease, sharing how his referral to the psychologist benefited him said: When the disease started, I became sad. I stayed in my room and cried...The team referred me to a psychologist who also warmly received me. She communicated well with me and gave me lots of advice to console me (Participant 4).

\section{Consultation setting}

A patient expressed concerns about lack of privacy in the following words: Hmm, the room is tight, because the room is small, some of my relatives had to wait outside. I could also see another doctor working in the same room, so I think the room was small. We could hear the other doctor and patient's conversation and I guess they could hear us as well....When it happens like that, it does not help. You do not have your privacy (Participant 4).

\section{Preferred model of care}

Some participants had been seen by the Palliative Care Team on an outpatient and home visit basis. Comparing the two services, some patients preferred home visits to outpatient consults. One participant said: I like the care that they provide me at home. I don't have many people who support me at home and anytime I come to the clinic I suffer. A day before I come to the clinic, I get worried and think about how I can make it there; because I cannot support my neck and I need to always lie down. Therefore, I will be thankful if they can always provide me care at home (Participant 1).

However, another participant felt he must come to see doctors at the hospital and not the other way round. He said: The team wants to help me by visiting me at home but I don't like that, I want to come to them. I am the sick one so I have to come to them to seek care (Participant 3).

\section{Cost effectiveness}

Participants generally felt their expenditure on healthcare had reduced although they felt better. One participant said: Eeii madam! Because of this same pain issue, they passed some medications through my veins so that my pain would be controlled and all the kind of laboratory investigations that I was made to do. I spent a lot, but here I don't spend much (Participant 4).

Another participant further buttressed the cost-effectiveness of palliative care as follows: Hmm, the drugs I bought, blood transfusions, I have spent a lot of money. I have spent money; aah I have been financially drained. However, since I came to the unit [palliative care], I have spent less (Participant 5).

\section{Process of care}

This theme discusses the perception of patients on how palliative care professionals carry out their care. Three (3) subthemes emerged: how they communicated with patients, involved them in decision making, and managed their symptoms.

\section{Communication}

Participants felt the palliative care team communicated well with them through their hospitality and reassurance, giving them 
adequate time to express their needs and concerns, and having access to the team's contact number. Referring to the therapeutic atmosphere established by the team, one participant said: When you are sick and you go to other facilities, the way you are treated can worsen your condition. How they [palliative care nurses] communicated with me has made me feel comforted (Participant 3).

An aspect of communication touched on by participants was the approach to reception used by the team. Participants felt welcome even on their first visit to the consulting room. One participant said: I was very happy about how they received me on the first day, as if they already knew me. Oh, I was warmly received by the doctor and the nurses (Participant 1).

Another participant who had developed confidence in the palliative care team and had renewed hope about her wellbeing due to their warm reception said, "I was warmly received. Since then I have been comforted. Because of them I will never give up" (Participant 2).

When participants were asked about how they were able to communicate with the palliative care team if they had any concerns, one participant said, "they gave me their contact number. I can call them whenever I have a question" (Participant 3).

Participants felt the palliative clinicians were very friendly and exuded empathy and kindliness to them. One participant said, "They have compassion and are hospitable to their patients" (Participant 2).

Most participants felt that the palliative care team listened to them. As a result, they are able to express their feelings and share concerns and issues that are important to them. One participant referred to this in these words, "they also asked me to feel free and tell them all my problems so that they will know how best to help me" (Participant 3).

Similarly, another participant said, "they take time to listen to all my concerns" (Participant 2). Further elaborating on the amount of time the team engages their patients in discussions, a participant stated, "On the first day, they spent almost one hour with me and asked me lots of questions" (Participant 3).

Participants further discussed the openness of the team and the chance they offer to patients to express their emotions.
I remember when I came there, the doctor said something and I will never forget. He said, "What I am going to discuss with you if you feel like crying, cry because there could be sadness. If you want to laugh, laugh, I give you the chance. Whatever you want to do, do it". Who am I that a doctor will tell me this? However, he gave me the chance to do what I like. Therefore, I can conclude that he is a man of God (Participant 3).

\section{Involvement in decision making}

Regarding the involvement of participants in decision making by the palliative care team, most of the participants indicated that the team solicited their views about their care. One participant said, "they ask me whatever they want to do for me. They ask for my view, then I tell them to do what they think will help me" (Participant 3).

\section{Symptom management}

Another aspect of the service offered by the palliative care team, which was highlighted by participants, is their attention to patients' symptoms and efforts to manage them. One of the participants referring to this, said, All my concerns were that I had stomach pain. I could not bear the pain after meals. I also vomited whenever I ate. ...They gave me some syrup which helped to control the pain. They also gave me tablets to control the vomiting (Participant 4).

\section{Discussion of Findings}

Participants in this study experienced the employment of a multidisciplinary team approach to care by the palliative care team, to meet specific health needs. Thus, some participants had been referred to other specialists to see patients, to get certain needs addressed or to seek their opinion in planning the care of patients. This finding is in accordance with a study by Masel., et al. (2016) which reported that patients who received care at a palliative care unit perceived their care as an interdisciplinary activity [10]. Similarly, a systematic review conducted by Virdun, Luckett, Davidson and Phillips (2015) found that one of the most important elements of end-of-life care identified by patients was expect care by the palliative care interdisciplinary team [11].

Cost effectiveness of palliative care is one factor that emerged from interaction with participants of the study. Participants felt they spent relatively less money after referral to the palliative care unit compared to other units they had been on. This finding is con- 
sistent with the findings made by May., et al. (2016) that, when palliative care consultation is provided within 2 days of admission, direct hospital cost decreases [12]. Key contributory factors to this trend include reduced frequency of re-hospitalization, avoidance of investigations that may not contribute to care provision, and avoidance of therapies that will play no role in enhancing the quality of life of the patient $[13,14]$.

Palliative care is delivered through various delivery models. Hence, depending on what is being practiced in an institution, the patient can make a choice for the place of care. The findings of the study revealed that some participants indicated preference for home care while others preferred care at the health facility for various reasons. This finding supports the findings of a study by Sandsdalen (2016), which revealed that some patients preferred to receive palliative care at home while others preferred care at the institutions [15]. In this study, those who preferred home care were of the view that receiving care in the comfort of their own home benefited them because of difficulty in accessing care at the health facility due to their poor health. The preference for receiving care in the comfort of patients' own home was similarly indicated by participants in a qualitative study published by Canadian Cancer Research Journal. However, in this previous study, participants preferred home-based palliative care because it helped them better interact with family and friends, further enhancing their sense of well-being [16].

In the current study, some participants expressed concerns about the need for privacy at the palliative care consulting rooms. These participants felt they could not express themselves during conversations with the team due to the presence of other patients in the environment in which they were seen. This finding established consistency with a qualitative study which sought to examine satisfaction with care in a palliative care unit, where patients and caregivers expressed the need for private rooms at palliative care units. One reason for the private rooms was the wish to have conversations with health care providers in private [17]. Similarly, the findings of a systematic review of patients' preferences in palliative care conducted by Sandsdalen, Hov, Høye, Rystedt, and Wildelarsson (2015) revealed that, participants preferred to receive palliative care in a more comfortable and relaxed environment [18].

Another significant finding emerging from this study was good communication by the palliative care team, which was expressed through warm reception and active listening. Participants felt that the palliative care team members took time to listen to them; hence, they were able to share concerns and issues that were important to them. This finding corroborates with a study by van Alsat (2013) in which patients and families felt the palliative care team listened to them [19].

Participants also mentioned that regular communication with the palliative care team benefited them. This was made possible because the team members made their contact numbers available to participants. This finding is inconsistent with the findings in a similar study conducted in Malawi, where participants shared their concern that the contact numbers of palliative care nurses were not made available for guidance and advice for support of caregivers [20].

The study further found that, participants were involved by clinicians in decision making concerning their care. Participants indicated that the palliative care team did not do whatever they wished for them but rather solicited their views about care. This finding is consistent with a survey conducted by Heins, Hofstede, and Rijken (2018) in which patients felt that palliative care home nurses always solicited their views and respected their decisions in providing care for them. Participants in both the previous study and the current study were appreciative of this approach [21].

Patients experiencing chronic illness suffer from many physical symptoms which affect their overall quality of life. Palliative focuses on relieving those symptoms to improve their quality of life. An essential prerequisite to good symptom management is carrying out an impeccable assessment of the patient [2]. Participants in this study highlighted that palliative care professionals took time to assess their symptoms to provide appropriate treatment. This finding is consistent with standards developed by the National Coalition for Hospice and Palliative Care (NCHPC). In their guideline document, the Clinical Practice Guidelines for Quality Palliative Care, the second domain of palliative care, discusses the assessment and management of physical symptoms as an important unavoidable aspect of palliative care [22].

\section{Conclusion}

The study shows that patients had overwhelming appreciation for excellent communication. This must be of great interest and attention to clinical educators, whose central role, among other 
things, is to enable clinicians under training, learn and assume the right attitude. Clinicians must also strategically position themselves in this regard and alter their behavior and attitudes to meet the expectations of patients and their families.

In view of the overwhelming appreciation of the care rendered by the palliative care team, clinicians can play various roles in advocating referral to specialist palliative care services. Secondly, clinicians must integrate the basic palliative care principles in their daily practice.

This study opens an avenue for future research on the caregiver perspective of palliative care as their perspective of what is important in care also represents an aspect of quality palliative care.

\section{Acknowledgement}

We are very grateful to the Faculty of the Ghana College of Nurses and Midwives, especially Dr. Prince Appiah-Yeboah, Rev. Mrs. Charity Essie Djokoto and Ms. Dzigbordi Kpikitse for their constructive review and comments, as well as the Palliative Care Teams of the Komfo Anokye Teaching Hospital and Korle Bu Teaching Hospital for their support and guidance throughout the study.

\section{Conflict of Interest}

The authors declare that there is no conflict of interest with respect to this study or its publication.

\section{Bibliography}

1. World Health Organization. "Noncommunicable diseases: Key facts". World Health Organization (2021). https://www.who. $\mathrm{int} /$ news-room/fact-sheets/detail/noncommunicable-diseases on September 14, 2021

2. World Health Organization. "Palliative Care: Key facts". World Health Organization (2020). https://www.who.int/newsroom/fact-sheets/detail/palliative-care

3. L Ford. "Palliative care unavailable for majority of patients in developing countries". Guard (2014): 2-4. https://www.theguardian.com/global-development/2014/jan/28/palliativecare-patients-developing-countries

4. MK Gyakobo., et al. "Developing a Model Palliative Care Korle Bu Palliative Care Team" (2015). https://www.sun.ac.za/ english/faculty/healthsciences/Family\%20Medicine $\% 20$
and\%20Primary\%20Care/PublishingImages/primafamedconference-2015-accra/Dr\%20Edwina\%200pare-Lokko\%20 (Ghana)\%20on\%20Developing\%20a\%20model\%20palliative $\% 20$ care $\% 20$ service $\% 20$ and $\% 20$ the $\% 20$ Korle-Bu\%20 experience.pdf

5. R Ofosu-Poku., et al. "Referral of patients with non-malignant chronic diseases to specialist palliative care: A study in a teaching hospital in Ghana". Int J Chronic (2017): 1-9. doi: $10.1155 / 2020 / 8432956$.

6. World Health Organization, "Quality of care: A Process for Making Strategic Choices in Health Systems". World Health Organization (2006).

7. S Ospina. “Qualitative Research,” Encyclopedia of Leadership. SAGE Publications (2004).

8. GD Shank. Qualitative Research: A personal Skills Approach. Prentice Hall (2002).

9. LM Given. "Lived Experience". The SAGE Encyclopaedia of Qualitative Research Methods (2008).

10. EK Masel., et al. "What Makes a Good Palliative Care Physician? A Qualitative Study about the Patient's Expectations and Needs when Being Admitted to a Palliative Care Unit". Plos one (2016): 1-10. doi: 10.1371/journal.pone.0158830

11. C Virdun., et al. "Dying in the hospital setting: A systematic review of quantitative studies identifying the elements of endof-life care that patients and their families rank as being most important". Palliative Medicine 29.9 (2015): 774-796.

12. P May., et al. "Palliative Care Teams' Cost-Saving Effect is Larger for Cancer Patients with Higher Numbers of Comorbidities," 35.1 (2016): 44-53. doi: 10.1377/hlthaff.2015.0752

13. MP Davis., et al. "A review of trials which examine early integration of outpatient and home palliative care for patients with serious illness". Annals of Palliative Medicine 4.3 (2015). doi: 10.3978/j.issn.2224-5820.2015.04.04

14. D Kavalieratos., et al. "Association Beween Palliative Care and Patient and Caregiver Outcomes: A Systematic Review and Meta-analysis". JAMA: The Journal of the American Medical Association 316.20 (2016). doi: 10.1001/jama.2016.16840. 
15. T Sandsdalen. "Quality in palliative care from the patient perspective Quality in palliative care from the patient perspective," (2016). https://www.diva-portal.org/smash/get/ diva2:1044098/FULLTEXT02.pdf

16. M Vierhout., et al. "The views of patients with brain cancer about palliative care: a qualitative study". Current Oncology 24.6 (2017): 1-12.

17. K Wentlandt., et al. "Quality of Care and Satisfaction With Care on Palliative Care Units". Journal of Pain and Symptom Management 51.2 (2016): 184-192.

18. T Sandsdalen., et al. "Patients' preferences in palliative care: A systematic mixed studies review". Palliative Medicine (2015): $1-21$.

19. DL Van Alsat. "The Important Thing is...: Patient and family voice in palliative care". University of Canterbury (2013).

20. E Mkwinda and E Lekalakala-Mokgele. "Palliative care needs in Malawi: Care received by people living with HIV". Curationis 39.1 (2016): 1664.

21. M Heins., et al. "Palliative care for patients with cancer: do patients receive the care they consider important? A survey study" (2018): 1-14.

22. National Consensus Project for Quality Palliative Care, Clinical Practice Guidelines for Quality Palliative Care, $4^{\text {th }}$ edition". National Coalition for Hospice and Palliative Care (2018).

\section{Volume 3 Issue 10 October 2021}

\section{(C) All rights are reserved by Gladys Anyane., et al.}

